\title{
Warum ist sein Blutzucker so schwer einzustellen?
}

\author{
Ein jugendlicher Patient aus Afghanistan wird mit einem entgleisten Blutzuckerspiegel \\ stationär aufgenommen. Bereits klinisch kommt der Verdacht auf, dass die Ursache seiner \\ Erkrankung nicht im Pankreas zu suchen ist. Labor und Bildgebung sichern die Diagnose.
}

Ein 16-jähriger afghanischer Jugendlicher wird mit einem Blutzucker von über $600 \mathrm{mg} / \mathrm{dl}$ notfallmäßig stationär eingewiesen. Bei der Aufnahme ist der Patient wach, es besteht keine Azidose, der Base-Exzess und das Serumnatrium sind normal. Der $\mathrm{HbA}_{1 \mathrm{c}}$-Wert liegt bei $15 \%$. Sonografisch und laborchemisch findet sich eine Fettleber, das Serumcholesterin beträgt $>350 \mathrm{mg} / \mathrm{dl}$ und das TSH ist erniedrigt. Auch unter Insulin lagen die BZ-Werte in der letzten Zeit nur selten unter $200 \mathrm{mg} / \mathrm{dl}$.

Bei der klinischen Untersuchung fallen grobe Gesichtszüge und große Akren auf. Die Körpergröße des Patienten liegt über der elterlichen Zielgröße. Der Patient klagt über Kopfschmerzen und Brennen an Fußsohlen und Handflächen. Am Kopf findet sich eine Narbe. Dort sei er vor Jahren in Afghanistan wegen eines Tumors operiert worden, aber man habe nicht alles entfernen können.

\section{MRT zeigt Tumor}

Bei Verdacht auf ein sezernierendes Hypophysenadenom wird eine kontinuierliche Messung des Wachstumshormons (GH) durchgeführt. Hierbei zeigt sich

\section{Fabula docet}

Bei einem schwer einstellbaren Diabetes ohne Neigung zur Azidose sollte mal auch einmal an ein GH-sezernierendes Hypophysenadenom denken, vor allem wenn eine Akromegalie besteht.

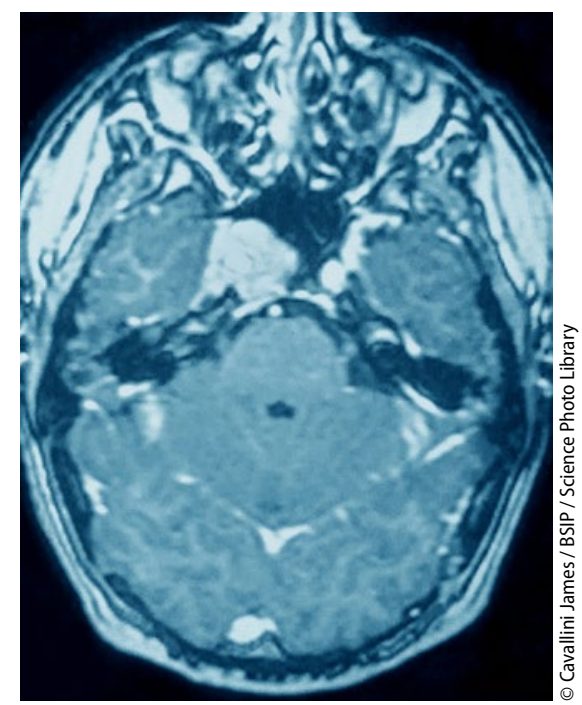

CT: ausgedehnter Hypophysentumor.

eine irreguläre, basal und pulsatil vermehrte GH-Sekretion. Das daraufhin veranlasste MRT zeigt eine tumoröse Raumforderung im Bereich der Hypophyse. „Es wurde ein erneuter operativer Eingriff durchgeführt, wobei aber wiederum nicht alles Tumorgewebe entfernt werden konnte, “ berichtete Dr. Ulrich Paetow von der Universitätskinderklinik in Frankfurt a. M.

\section{Leitsymptom Akromegalie}

Die vermehrte Bildung von $\mathrm{GH}$ bei einem sezernierenden Hypophysenadenom führt typischerweise zu einer Akromegalie bzw. zu Gigantismus. Lokale Symptome sind Kopfschmerzen und Sehstörungen. Bei ca. $80 \%$ der betroffenen Patienten entwickelt sich auch ein Hypogonadismus bzw. eine Hypophysenvorder-
lappen-Insuffizienz. Bei jedem Zweiten besteht eine gestörte Glukosetoleranz und jeder Fünfte ist Diabetiker. An der Haut kann sich eine Acanthosis nigricans entwickeln. Meist finden sich auch EKGVeränderungen wie eine Verlängerung der QTc-Zeit. Weitere Komplikationen sind obstruktives Schlaf-Apnoe-Syndrom, Hypertonie, Myopathie und Karpaltunnelsyndrom.

\section{Unterschiedliche GH-Wirkungen}

Beim Gesunden zeigt GH ein dem Insulin gegenläufiges Sekretionsmuster. Bei einem akuten Exzess wirkt GH insulinähnlich, bei einer chronisch vermehrten Sekretion aber insulinantagonistisch, wobei die zelluläre Glukoseverwertung und die Insulinsekretion der Betazelle gestört werden. „Hoch dosierte langfristige $\mathrm{GH}-\mathrm{Exp}$ ositionen erzeugen einen permanenten Diabetes mit schlechter Rückbildungstendenz", so Paetow.

So besteht auch nach der Rezidivoperation bei diesem Patienten der Diabetes weiter, wobei die Insulinresistenz im Vordergrund steht. Neben der antidiabetischen Therapie mit Insulin, Metformin und GLP-1-Analogon wird auch eine Therapie mit einem Somatinanalogon, was allerdings die Insulinausschüttung hemmt und damit eine Hyperglykämie induzieren kann, dem Dopaminagonisten Cabergolin und dem Antiöstrogen Tamoxifen eingeleitet. Die beiden letzteren hemmen die GH-Wirkung. Diskutiert wird auch eine Radiotherapie.

Dr. med. Peter Stiefelhagen

- Fallpräsentation von Dr. Ulrich Paetow, Frankfurt a.M., im Rahmen der Herbsttagung der DDG, 11. 11. 2016 in Nürnberg 